\title{
Variability of halo carbon stars ${ }^{\star}$
}

\author{
P. Battinelli ${ }^{1}$ and S. Demers ${ }^{2}$ \\ 1 INAF, Osservatorio Astronomico di Roma Viale del Parco Mellini 84, 00136 Roma, Italy \\ e-mail: battinel@inaf.it \\ 2 Département de Physique, Université de Montréal, CP 6128, Succursale Centre-Ville, Montréal, Qc H3C 3J7, Canada \\ e-mail: demers@astro.umontreal.ca
}

Received 24 February 2012 / Accepted 7 June 2012

\section{ABSTRACT}

\begin{abstract}
Context. Carbon stars are among the brightest intermediate-age stars. Over one hundred have been identified in the Galactic halo. Since the halo consists essentially in an old stellar population, we believe that these $\mathrm{C}$ stars are trespassers and belong to streams left over by disrupted dwarf spheroidal galaxies.

Aims. By performing photometric monitoring we intend to detect long-period variables among halo carbon stars. We should be in position to identify Mira, semi-regular, and irregular variables and determine their period and age group.

Methods. We obtained, over several semesters, $K, J$, and $I$ images centered on the $\mathrm{C}$ stars in order to determine their variation and periodicity.

Results. We establish the period of 14 program stars and discover 13 Miras among them. Most of them belong to the 1-3 Gyr age group.

Conclusions. The period distribution of the halo Miras closely matches that of the Miras of Fornax. The lack of old Miras suggests that the majority of the halo Miras likely do not originate from the Sagittarius dwarf spheroidal galaxy, which is believed to be older than 5 Gyr.
\end{abstract}

Key words. stars: AGB and post-AGB - stars: carbon - stars: variables: general - Galaxy: halo

\section{Introduction}

Carbon stars represent the brightest members of the intermediate-age population. Their evolutionary status and age were established by Catchpole \& Feast (1973), who observed a few intermediate-age globular clusters in the Small Magellanic Cloud containing carbon stars. They are also seen in massive dwarf spheroidal galaxies, such as Fornax (Demers \& Kunkel 1979; Aaronson \& Mould 1980), Leo I (Lee et al. 1993), or Sagittarius (Sgr) (Whitelock et al. 1999).

Carbon stars are not found in Galactic globular clusters. In principle, they should also not be seen in the halo of the Galaxy, which is made up of old stars. However, extremely red stars, observed at high Galactic latitudes, were confirmed to be C stars by Totten $\&$ Irwin (1998), and subsequently, over one hundred halo C stars, selected from their 2MASS colours, were spectroscopically confirmed by Mauron et al. $(2004,2005,2007)$ and Mauron (2008).

If we understand the star formation history of the Galactic halo sufficiently well, then these stars must be alien trespassers. Some of them are faint enough to suggest that they have distances from the Sun of greater than $50 \mathrm{kpc}$. The Totten \& Irwin (1998) carbon star survey led Ibata et al. (2001) to trace, for the first time, the tidal stream of the Sagittarius dwarf spheroidal galaxy orbiting the Galaxy. Ibata et al. (2001) concluded, however, that roughly half of the halo carbon stars, known at the time, do not seem to belong to the Sgr stream. These stars could belong to some other unidentified streams, although this has not yet been established. Accurate distances and velocities would be required to assess their origin more accurately.

\footnotetext{
* Based on observations made with the REM Telescope, INAF Chile.
}

It is known that the majority of carbon stars are variable. All the $\mathrm{R}$ and $\mathrm{N}$-type $\mathrm{C}$ stars observed by Hipparcos indeed, turned out to be either irregular, semi-regular, or nearly periodic variables (Grenon et al. 2000). CH stars originate from mass transfer in binary systems and with colours $(J-K)<0.8$ (Goswami et al. 2010) they form a class of objects photometrically distinct from our N-type stars, which have $(J-K)>1.4$. Since C stars are thin-disk objects, variable star surveys have been limited to low Galactic latitudes. However, two high Galactic latitude C star variables were detected by Meusinger \& Brunzendorf (2001) and Meusinger (2002), which are both close to the direction of the globular cluster M92. One with a 2 MASS $(J-K)=1.35$ has a period of $\sim 0.81 \mathrm{yr}$, while the second one is bluer, $(J-K)=$ 0.67 , and has no known period.

Evidence has accumulated suggesting that the period of a Mira, regardless of whether it is O-rich or C-rich, is related to its mass and age. These clues were briefly summarized by Feast (2009). Evidence has come from the kinematics of Miras around the Sun (Feast et al. 2006) and the study of LMC globular clusters, of known age, containing Miras (Nishida et al. 2000; van Loon et al. 2003; Kamath et al. 2010). Periods of Miras in dwarf spheroidal galaxies are also compatible with the known star formation history of these galaxies (Menzies et al. 2011). Essentially, low-mass old giants have short periods while young ones ( $<1$ Gyr) have periods, well over 500 days. Thus, the detection of the variability and a period determination of a halo $\mathrm{C}$ star provide important clues to their origin.

A more clearly defined age for the halo $\mathrm{C}$ stars would provide insights into the merging history of the hypothetical "victim" that left its debris around our Galaxy. The recent identification of a group of younger globular clusters by the HST ACS Survey, led to similar conclusion. It is indeed very tempting to argue 
Table 1. id numbers, coordinates and $K_{\mathrm{s}}$ magnitudes of selected targets.

\begin{tabular}{lccccc}
\hline \hline \multirow{2}{*}{ Id } & \multirow{2}{*}{ RA } & Dec & $\ell$ & $b$ & $K_{\mathrm{s}}$ \\
\hline $\mathrm{m} 31$ & $00: 16: 55.8$ & $-44: 00: 40.6$ & 323.1 & -71.7 & 7.1 \\
m84 & $00: 36: 32.3$ & $-22: 54: 51.0$ & 83.4 & -84.6 & 14.1 \\
m35 & $08: 59: 55.7$ & $-77: 53: 05.4$ & 292.0 & -20.3 & 10.5 \\
m06 & $11: 09: 59.7$ & $-21: 22: 01.1$ & 273.5 & +35.6 & 7.0 \\
m37 & $11: 41: 42.4$ & $-33: 41: 33.2$ & 286.7 & +27.0 & 12.2 \\
m41 & $13: 47: 23.0$ & $-34: 47: 23.3$ & 315.8 & +26.7 & 10.1 \\
m11 & $13: 59: 20.6$ & $-30: 23: 39.4$ & 319.9 & +30.2 & 11.8 \\
m48 & $18: 46: 50.3$ & $-56: 14: 02.8$ & 339.6 & -21.7 & 11.0 \\
m49 & $19: 14: 24.2$ & $-78: 22: 41.5$ & 316.0 & -27.7 & 7.4 \\
m50 & $19: 31: 38.5$ & $-30: 02: 30.5$ & 9.1 & -21.3 & 10.3 \\
m51 & $19: 37: 09.8$ & $-35: 30: 14.9$ & 3.9 & -24.1 & 10.2 \\
m52 & $19: 37: 34.1$ & $-35: 32: 37.6$ & 3.9 & -24.2 & 9.1 \\
m16 & $19: 42: 19.0$ & $-35: 19: 37.6$ & 4.4 & -25.1 & 10.1 \\
m17 & $19: 42: 21.3$ & $-32: 11: 04.1$ & 7.7 & -24.1 & 10.0 \\
m18 & $19: 48: 50 ; 6$ & $-30: 58: 31.9$ & 9.4 & -25.1 & 9.8 \\
m19 & $19: 53: 30.2$ & $-38: 35: 59.4$ & 1.5 & -28.1 & 9.2 \\
m20 & $20: 13: 19.4$ & $-23: 41: 44.2$ & 19.1 & -27.9 & 8.1 \\
m22 & $20: 54: 54.5$ & $-28: 28: 56.7$ & 16.8 & -38.2 & 10.8 \\
m24 & $22: 06: 53.7$ & $-25: 06: 28.2$ & 26.6 & -53.2 & 8.9 \\
m25 & $22: 17: 09.9$ & $-26: 07: 03.3$ & 25.6 & -55.6 & 8.9 \\
m53 & $22: 43: 50.3$ & $-57: 01: 23.3$ & 331.1 & -52.5 & 8.0 \\
m54 & $22: 46: 28.5$ & $-27: 26: 58.2$ & 25.0 & -62.3 & 13.3 \\
m55 & $22: 47: 38.0$ & $-78: 27: 17.2$ & 310.3 & -36.8 & 8.2 \\
m26 & $23: 17: 21.1$ & $-24: 11: 42.4$ & 35.5 & -68.6 & 12.3 \\
m28 & $23: 25: 31.4$ & $-30: 10: 56.0$ & 18.6 & -70.9 & 11.0 \\
\hline & & & & &
\end{tabular}

that their origin is related to their formation within Milky Way satellite galaxies that were later accreted (Marin-Franch et al. 2009).

\section{Targets and observations}

The 25 carbon stars selected for monitoring are given in Table 1. The id numbers are from Mauron's lists. We list their equatorial J2000.0 coordinates and both their Galactic longitude and latitude along with their $K_{\mathrm{s}}$ magnitude from 2MASS. Since they were to be observed from Chile, we selected targets with $\delta<-20^{\circ}$ in order to be able to observe them over several months. The observations, presented in this paper, were secured with rapid eye mount (REM), which is a robotic $60 \mathrm{~cm}$ telescope located on La Silla. The telescope hosts two instruments: REMIR, an infrared imaging camera with a $512 \times 512$ array giving a scale of $1.221^{\prime \prime}$ per pixel, and ROSS, a visible imager, equipped with a $1024 \times 1024 \mathrm{CCD}$ giving a scale of $0.575^{\prime \prime}$ per pixel. The two cameras can observe simultaneously thanks to a dichroic placed before the telescope focus. The field of view is $10^{\prime} \times 10^{\prime}$. The observatory is operated for INAF by the REM Team ${ }^{1}$.

Observations were obtained, every $\sim 15$ days, weather permitting, during the long observing season of each target. Targets were followed during four semesters, from February 2010 to January 2012. One observation consists of two $I$ exposures of $60 \mathrm{~s}$ and two $K^{\prime}$ and $J$ exposures of $30 \mathrm{~s}$ each. The images were analysed with SExtractor (Bertin \& Arnouts 1996). Since the differences between $K^{\prime}$ and $K_{\mathrm{S}}$ are of the order of a few hundredths, we omit the prime and the "s".

The NIR instrumental magnitudes are calibrated with 2MASS point source observations seen in the field. The $I$ images are calibrated with DENIS (Epchtein et al. 1997) data, which,

\footnotetext{
${ }^{1}$ http://www.rem.inaf.it
}

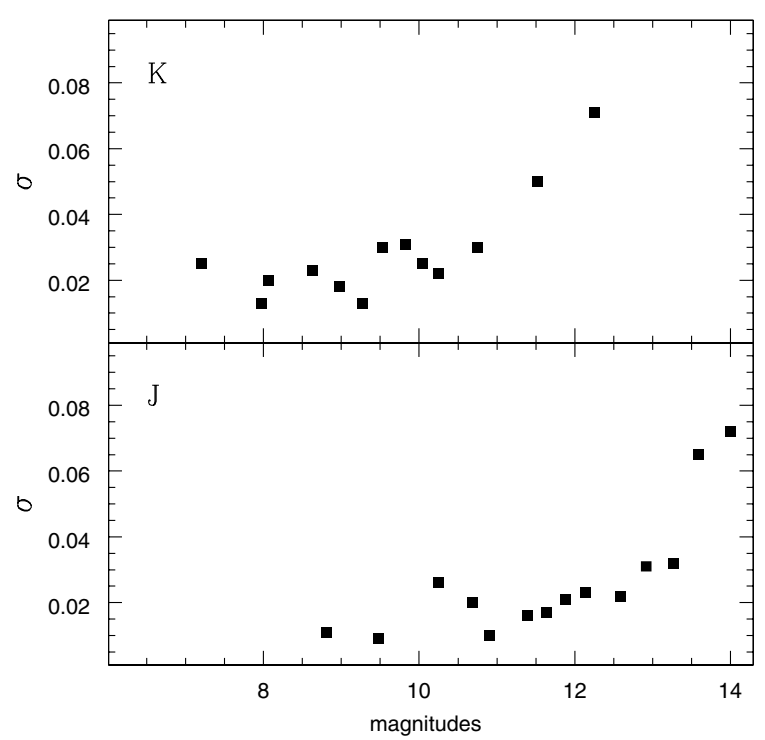

Fig. 1. Standard deviations of two observations as a function of magnitude. Each point represents the mean of eight measures.

Table 2. Mean magnitudes and colour of program stars.

\begin{tabular}{lcccccccc}
\hline \hline $\mathrm{Id}$ & $\langle K\rangle$ & $\langle J\rangle$ & $n_{K}$ & $n_{J}$ & $\langle J\rangle-\langle K\rangle$ & $\langle I\rangle$ & $n_{I}$ & $E(J-K)$ \\
\hline $\mathrm{m} 06$ & 7.16 & 8.73 & 25 & 25 & 1.60 & 10.72 & 18 & 0.02 \\
$\mathrm{~m} 11$ & 12.35 & 15.37 & 10 & 1 & & & & 0.03 \\
$\mathrm{~m} 16$ & 9.65 & 12.15 & 18 & 18 & 2.50 & & & 0.14 \\
$\mathrm{~m} 17$ & 9.60 & 11.65 & 17 & 17 & 2.05 & & & 0.07 \\
$\mathrm{~m} 18$ & 10.00 & 13.76 & 17 & 17 & 3.76 & & & 0.09 \\
$\mathrm{~m} 19$ & 9.25 & 11.50 & 20 & 20 & 2.25 & 13.28 & 17 & 0.04 \\
$\mathrm{~m} 20$ & 8.01 & 9.45 & 18 & 18 & 1.46 & & & 0.07 \\
$\mathrm{~m} 22$ & 10.99 & 12.49 & 18 & 17 & 1.50 & 14.30 & 11 & 0.05 \\
$\mathrm{~m} 24$ & 8.77 & 10.85 & 17 & 18 & 2.08 & 12.32 & 18 & 0.02 \\
$\mathrm{~m} 25$ & 8.76 & 10.75 & 17 & 18 & 1.99 & 12.39 & 17 & 0.01 \\
$\mathrm{~m} 26$ & 12.25 & 13.36 & 8 & 16 & 1.11 & & & 0.01 \\
$\mathrm{~m} 28$ & 10.16 & 12.74 & 14 & 18 & 2.58 & & & 0.01 \\
$\mathrm{~m} 31$ & 7.29 & 10.09 & 7 & 22 & 2.81 & 12.49 & 22 & 0.00 \\
$\mathrm{~m} 35$ & 10.45 & 12.48 & 27 & 26 & 2.03 & 14.24 & 13 & 0.10 \\
$\mathrm{~m} 37$ & 12.07 & 13.72 & 15 & 19 & 1.65 & & & 0.04 \\
$\mathrm{~m} 41$ & 9.96 & 11.80 & 24 & 24 & 1.84 & 13.46 & 16 & 0.03 \\
$\mathrm{~m} 48$ & 11.03 & 13.28 & 25 & 25 & 2.25 & 14.90 & 14 & 0.05 \\
$\mathrm{~m} 49$ & 7.83 & 10.27 & 28 & 29 & 2.44 & 12.82 & 27 & 0.11 \\
$\mathrm{~m} 50$ & 10.17 & 11.83 & 23 & 23 & 1.65 & 13.52 & 19 & 0.06 \\
$\mathrm{~m} 51$ & 10.07 & 11.72 & 24 & 24 & 1.65 & & & 0.15 \\
$\mathrm{~m} 52$ & 8.90 & 10.58 & 22 & 24 & 1.69 & & & 0.15 \\
$\mathrm{~m} 53$ & 7.98 & 9.37 & 23 & 22 & 1.39 & 10.93 & 23 & 0.01 \\
$\mathrm{~m} 54$ & 13.22 & 14.53 & 1 & 2 & 1.31 & & & 0.01 \\
$\mathrm{~m} 55$ & 7.85 & 9.60 & 20 & 27 & 1.75 & 11.24 & 27 & 0.08 \\
\hline
\end{tabular}

unfortunately, are not available for every field. The photometric accuracy in $J$ and $K$ depends on the apparent magnitudes, as shown in Fig. 1. We have calculated the standard deviation in two calibrated $J$ and $K$ measures obtained on the same night. The means of the two measures were sorted by brightness and binned into groups of eight. We plot the average standard deviation versus the average magnitude in each bin. We see that for $K>12$. and $J>13.4$ the photometric errors become significant.

\section{Results}

In Table 2, we present the arithmetic mean magnitudes and colours of our target stars. The numbers of observations in each 
P. Battinelli and S. Demers: Variability of halo carbon stars
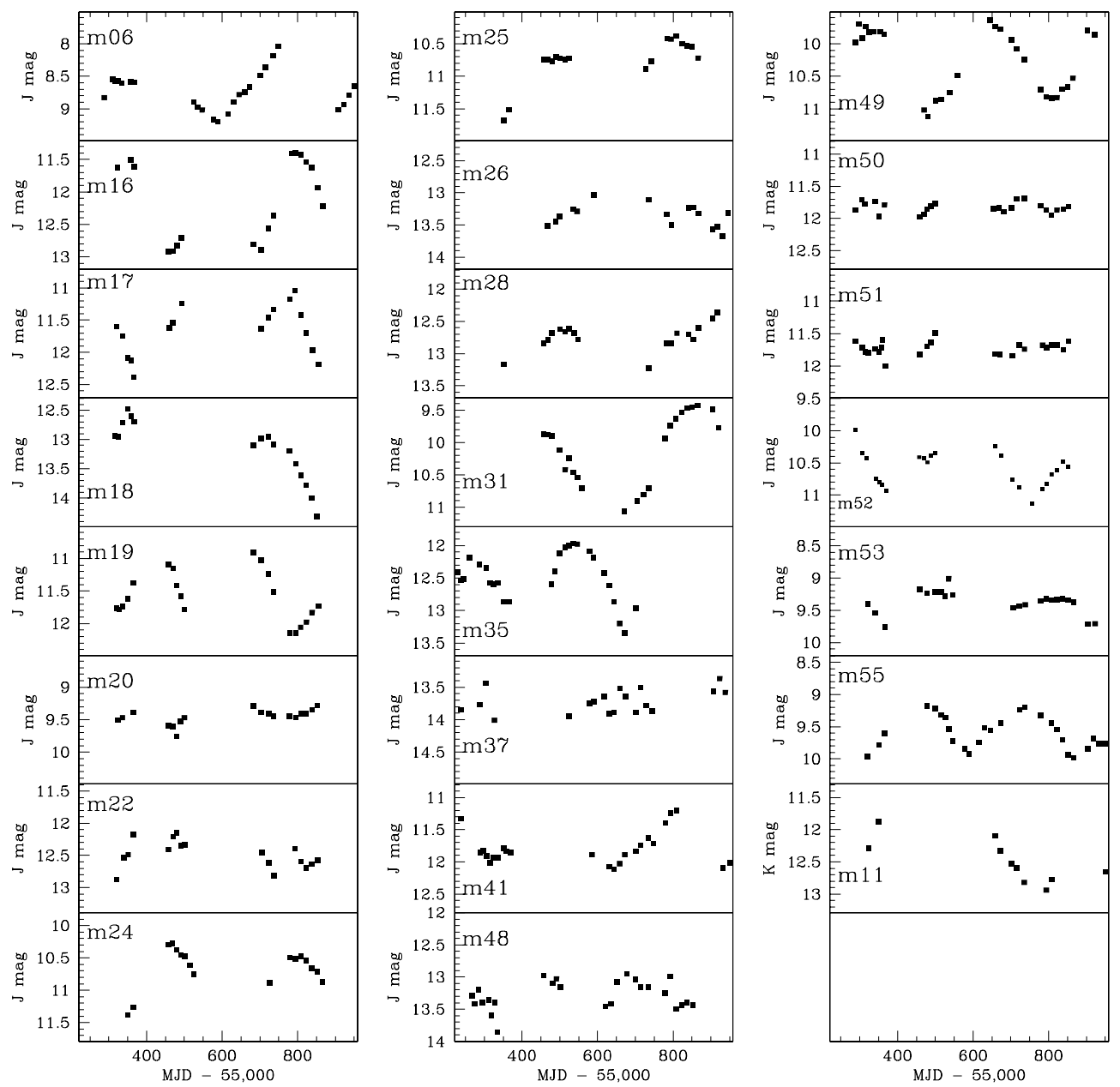

Fig. 2. $J$ magnitude variations of the 23 program stars. Note that the magnitude axis covers two magnitudes. For m11, we plot the $K$ mag.

band are also listed. The small number of $I$ magnitudes precludes their use for a period search. Stars with missing I magnitudes are those without DENIS stars and $I$ magnitudes in their field. We also include the reddening for $(J-K)$ taken from Schlegel et al. (1998) and adopt their transformations $E(J-K)=0.526 E(B-V)$ and $A_{K}=0.367 E(B-V)$. We note that for some targets, seen toward the Galactic centre, the reddening is not negligible. Three of our program stars, listed in Table 1, are too faint to be properly observed with the $60 \mathrm{~cm}$ telescope. $\mathrm{m} 84$ was withdrawn from the observing program because it was too faint; $\mathrm{m} 11$ is very red, 2MASS colour $\left(J-K_{\mathrm{s}}\right)=2.79$, and we obtained only one $J$ magnitude value which we give in Table $2 ;$ m54 is quite faint and often invisible, even when the two combined K 30 s exposures are used. We have few observations of these last two targets.

\subsection{Light variations}

The $J$ magnitudes of the program stars are plotted as a function of time in Fig. 2. To facilitate the amplitude comparison, the $Y$ axis covers two magnitudes in $J$, excepted for $\mathrm{m} 18$, which has a $J$ amplitude larger than 2 mag. For $\mathrm{m} 11$, we plot $K$ magnitudes.

Inspection of the figures reveals three types of variations: a large amplitude as in the case of $\mathrm{m} 18$; low amplitude as for $\mathrm{m} 50$, and a very irregular variation as for $\mathrm{m} 37$. The light variations of faint stars such as $\mathrm{m} 37, \mathrm{~m} 48$ and $\mathrm{m} 26$ display a large scatter.

To enable us to classify the low-amplitude variables, we inspected their $I$ mag observations, when available. The $I$ magnitudes of five of these stars are shown in Fig. 3. The inspection of the I mag variations neither reveals any periodicity nor provides significant additional clues about the nature of the stars.

\subsection{Period search}

We selected the $J$ magnitudes to search for periodicity because the variables have a larger amplitude in this band than in the $K$ band. $I$ magnitudes might have been useful in this respect but we have fewer $I$ measures because we started to obtain $I$ observations only during the second semester. A simple sine curve is fitted to the data points. The quality of the fit is defined by the $\chi^{2}$ parameter, which corresponds essentially to the mean squared deviation between the observed points and the fitted sine curve. We adopted the period yielding the smallest $\chi^{2}$. We are confident that the accuracy of the adopted periods is smaller than 10 days. Periods are seen to fluctuate within this range as we add a new data point. A second method was also attempted, the Phase Dispersion Minimization code (Stellingwerf 1978). Periods found with this approach did not produce better fits to the light curves. We are unable of course to determine periods longer than the time interval covered by our observations. We see no long secular trends, which would suggest periods of $600+$ days. In Table 3, we give the time interval (days) covered by the observations $(\Delta T)$, the periods found in days, the $\chi^{2}$ parameter indicating the quality of the sine fit, and the $K, J$, and $I$ amplitudes of the light curve along with remarks or classification. Even for genuine large-amplitude variables, the $\chi^{2}$ can be large if the light curve does not repeat itself from cycle to cycle. 


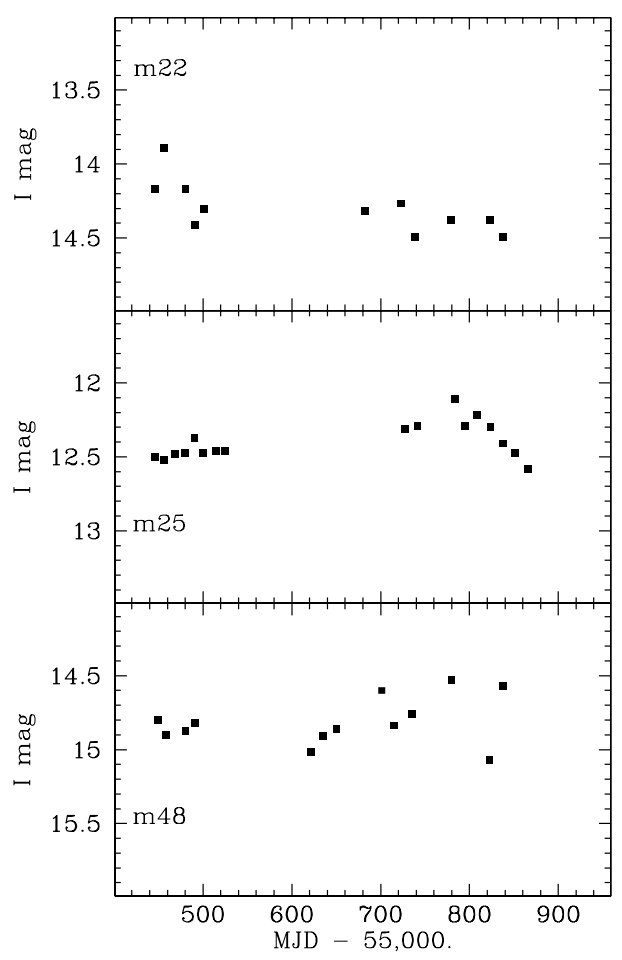

Fig. 3. I magnitude light variations for irregular or low-amplitude stars.

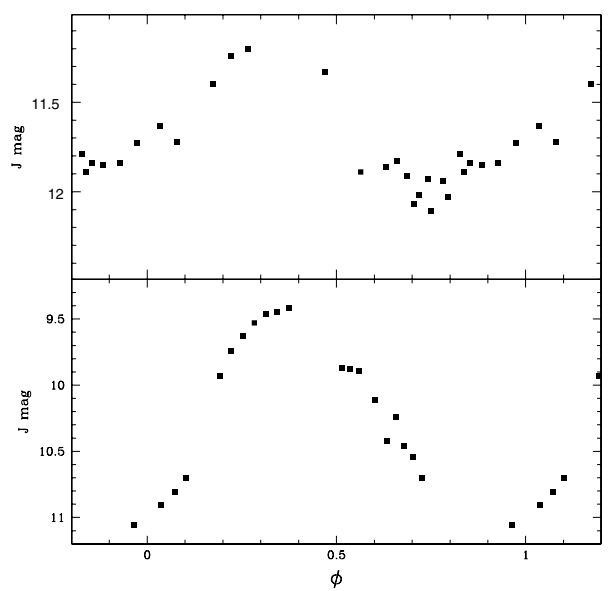

Fig. 4. Examples of light curves with different $\chi^{2}, 0.15$ for $\mathrm{m} 41$ (top) and 0.04 for $\mathrm{m} 31$ (bottom).

This is the case for both $\mathrm{m} 06$ also $\mathrm{m} 35$, which have two maxima that are not at the same luminosity. As a rule, we can says that if $\chi^{2}$ is greater than $\approx 0.15$ the light curve shows a substantial amount of scatter.

Soszyński et al. (2009) used the I-band amplitude to distinguish Miras and semi-regular variables. Miras are defined as stars with an $I$-band amplitude larger than 0.8 mag, while Whitelock (2012) also takes into account the $K$ amplitude, $\Delta K>0.4$ mag. On the basis of this criterion, we have 14 Miras in our sample. We include $\mathrm{m} 24$ in this class even if its $\Delta I$ is quoted as 0.6 mag because we do not have $I$ observations for the dates of the first $J$ magnitudes (see Fig. 2). Slightly more than $60 \%$ of the observed $\mathrm{C}$ stars are found to be Miras. In Fig. 4, we show two examples of light curves plotted against the phases. We note that the $x$ axis covers more than one cycle, some of the points being repeated.

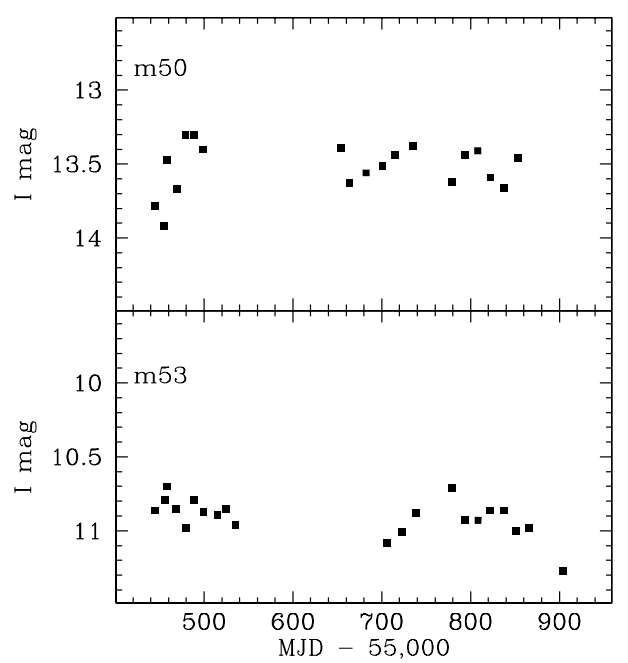

Table 3. Period, amplitude, and classification.

\begin{tabular}{lccccccc}
\hline \hline Id & $\Delta T$ & $P$ & $\chi^{2}$ & $\Delta K$ & $\Delta J$ & $\Delta I$ & Comment \\
\hline $\mathrm{m} 06$ & 663 & 332 & 0.21 & 0.9 & 1.1 & 1.5 & Mira, semiregular \\
m11 & 628 & 260 & 0.12 & 1.0 & & & Mira \\
m16 & 542 & 229 & 0.01 & 0.9 & 1.5 & Mira \\
m17 & 535 & 247 & 0.04 & 0.6 & 1.4 & Mira \\
m18 & 548 & 382 & 0.05 & 1.0 & 2.3 & & Mira \\
m19 & 535 & 256 & 0.04 & 0.7 & 1.2 & 1.6 & Mira \\
m20 & 529 & & & 0.2 & 0.5 & & low amplitude irregular \\
m22 & 531 & & & 0.6 & 0.7 & 0.6 & irregular \\
m24 & 515 & 327 & 0.03 & 0.6 & 1.0 & 0.6 & Mira? See text \\
m25 & 514 & & & & 1.2 & 0.4 & irregular \\
m26 & 478 & & & 0.2 & 0.5 & & low amplitude irregular \\
m28 & 567 & 361 & 0.27 & 0.3 & 0.6 & & low amplitude semiregular \\
m31 & 462 & 465 & 0.04 & 0.7 & 1.6 & 1.9 & Mira, $P \approx \Delta T$ \\
m35 & 471 & 277 & 0.20 & 0.9 & 1.3 & 1.3 & Mira \\
m37 & 701 & & & 0.4 & 0.4 & & irregular \\
m41 & 713 & 309 & 0.15 & 0.5 & 1.1 & 1.3 & Mira \\
m48 & 615 & & & 0.6 & 0.6 & 0.5 & faint, large scatter \\
m49 & 635 & 312 & 0.04 & 1.1 & 1.5 & 1.6 & Mira \\
m50 & 565 & & & 0.3 & 0.3 & 0.6 & very low amplitude, irregular \\
m51 & 564 & & & 0.3 & 0.5 & & low amplitude irregular \\
m52 & 563 & 358 & 0.07 & 0.5 & 1.2 & & Mira \\
m53 & 602 & & & 0.4 & 0.8 & 0.3 & irregular \\
m54 & 66 & & & & & & Mira \\
m55 & 629 & 276 & 0.13 & 0.6 & 0.8 & 1.0 & \\
\hline
\end{tabular}

\section{Discussion}

It is well-established that the period of a Mira represents a good indicator of the stellar population to which it belongs. Long-period Miras are expected to have high mass progenitors, thus belong to a younger population (Iben \& Renzini 1983). Therefore, the period of a Mira can be used as a proxy of its age, as suggested by Habing \& Whitelock (2004). For example, Feast et al. (2006) inferred, from the velocity dispersion of Galactic 


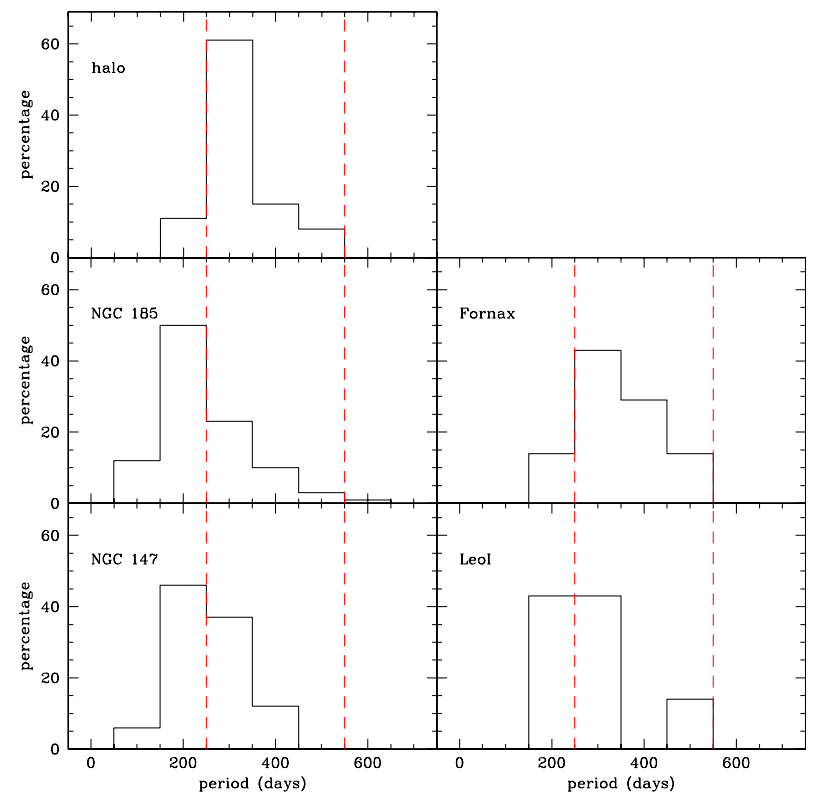

Fig. 5. Comparison of the period distributions of Miras. The dashed lines define the three age groups.

Miras, that those with periods longer than 500 days are $\approx 1 \mathrm{Gyr}$ old, while those with $P \approx 375$ days are $\sim 3$ Gyr. Hereafter, we adopt the Habing \& Whitelock (2004) period/age groups: Miras with $P<250$ days are older than $\sim 5$ Gyr; variables with periods between 250 days and 550 days are of intermediate-age, between 1 and $3 \mathrm{Gyr}$; longer periods correspond to Miras of $1 \mathrm{Gyr}$ old or younger.

The effect of metallicity on the period of a Mira is at the present time somewhat uncertain: very little has been done to investigate this relationship. Using oxygen-rich Miras and semiregular variables in globular clusters, Feast \& Whitelock (2000) established a "maximum period" versus metallicity relation. Since globular cluster stars are old, 96\% of Feast \& Whitelock (2000) sample have $P<250$ days. We do not know whether the trend extends to longer periods and whether carbon-rich variables also follow such a trend. We are therefore obliged to neglect the metallicity effect for the time being.

In Fig. 5, we compare the period distribution of the halo Miras with Miras in dwarf galaxies without current star formation. Data for the dwarf spheroidal galaxies are for Leo I, from Menzies et al. (2010), Fornax, from Whitelock et al. (2009), and both NGC 185 and NGC 147, from Lorenz et al. (2011), where we consider as Miras all variables with $\Delta I>0.8$.

The bulk of the Miras in NGC 185 and, to a lesser extent, NGC 147 are old stars, while most of the Miras in the Galactic halo and Fornax are of intermediate ages. In Leo I, about 60\% of the Miras are of intermediate-age; since there are currently only seven known Miras in this galaxy, its age distribution is poorly defined. Fornax has had a long and complex star formation history. Intermediate-age stars are abundant in its inner and intermediate fields (Held et al. 2009), which suggests that the C star Miras located in the Galactic halo originate from a population somewhat similar to Fornax. The Sgr galaxy could be a possible candidate. Unfortunately, its Mira population has not yet been surveyed, but Bellazzini et al. (2006) estimated that the mean age of its central population is $8 \mathrm{Gyr}$. We should then expect its period distribution to differ from that of Fornax, and for it to contain many short-period Miras.
Table 4. Distances of Miras.

\begin{tabular}{lccc}
\hline \hline Id & $D(\mathrm{kpc})$ & $\mathrm{id}$ & $D(\mathrm{kpc})$ \\
\hline $\mathrm{m} 06$ & 10.6 & $\mathrm{~m} 24$ & 19.9 \\
$\mathrm{~m} 11$ & 78.2 & $\mathrm{~m} 31$ & 11.4 \\
$\mathrm{~m} 16$ & 21.2 & $\mathrm{~m} 35$ & 39.0 \\
$\mathrm{~m} 17$ & 24.8 & $\mathrm{~m} 41$ & 35.4 \\
$\mathrm{~m} 18$ & 25.8 & $\mathrm{~m} 49$ & 12.1 \\
$\mathrm{~m} 19$ & 21.4 & $\mathrm{~m} 52$ & 22.9 \\
& & $\mathrm{~m} 55$ & 11.9 \\
\hline
\end{tabular}

\subsection{Distances of Miras}

It has been known for more than 30 years (Glass \& Lloyd Evans 1981) that Mira variables, discovered by optical surveys, follow a period-luminosity relation in the near infrared. However, surveys performed in the near-infrared also identify dusty variables with circumstellar envelopes that are missed by optical surveys. The dust that affects the $K$ magnitudes is responsible for the large scatter seen in the $K-\log P$ plane, as in the case, for example, of the Miras in NGC 6822 (Battinelli \& Demers 2011) observed during a survey done in the near-infrared.

To properly determine the $M_{K}$ of the Miras, regardless of whether they are dusty, we adopted the approach of Ita \& Matsunaga (2011) in using a P-L-C relation. Their colour correction was given as:

$$
K_{\text {obs }}-K_{\mathrm{PL}}=0.213(J-K)^{2}-0.586(J-K)+0.331 .
$$

We used the $\mathrm{M}_{K}$ period-luminosity relation of LMC carbon stars determined by Whitelock et al. (2008), but instead of adopting their LMC distance of 18.39 we used a modulus of 18.50 (Koerwer 2009; Pietrzyński et al. 2009; Laney et al. 2012):

$M_{K}=-3.52[\log P-2.38]-7.35$.

In Table 4, we list the estimated distances of the 14 Miras identified.

\subsection{Association with either the Sagittarius galaxy or stream}

Only one of our target stars, m50, is clearly associated with the Sgr galaxy. It is within its elongated central region, eight degrees from its centre. However, 4 other $\mathrm{C}$ stars are probably members of the "southern arc" stream, which was identified by Majewski et al. (2003), namely $\mathrm{m} 17, \mathrm{~m} 18, \mathrm{~m} 20$, and $\mathrm{m} 22$. Both $\mathrm{m} 17$ and $\mathrm{m} 18$ are Miras and their calculated distances closely match the Sgr distance of $24.8 \mathrm{kpc}$ determined by Kunder \& Chaboyer (2009). However, none of our targets are seen toward the Sgr stream in the southern hemisphere, $\ell \sim 160^{\circ}$ and $b \sim-50^{\circ}$, mapped by Koposov et al. (2012). This lack of association is not too surprising since Ibata et al. (2001) deduced that only half of the Totten \& Irwin (1998) halo C stars belong to the Sgr Stream. Since our list includes none of the Totten \& Irwin (1998) halo C stars, it represents an independent sample. Whitelock et al. (1999) estimated that the Sgr galaxy contains at least $100 \mathrm{C}$ stars. They mention that several C stars have large amplitude variations and are probably Miras. However, only 4 of them have known periods.

If the bulk of the Sgr stars were indeed older than $5 \mathrm{Gyr}$ (Bellazzini et al. 2006), then most of the Miras associated with Sgr should have periods shorter than 250 days. Unfortunately, any analysis at present is strongly affected by small number statistics. Four Sgr Miras have periods determined by Whitelock et al. (1999), only one of which has a $P<250$ days. From Fig. 5, 
we see that barely two of the 13 newly discovered halo Miras have such a short period. Therefore, we tentatively propose that the majority of the halo Miras may have an origin that is unrelated to Sgr. We are conducting, however, an ongoing survey of the Sgr carbon stars designed to determine whether they do indeed have a short-period excess, as implied by most of their ages. We are currently surveying a second sample of halo $\mathrm{C}$ stars to enhance our data set for the analysis of the period distributions.

Acknowledgements. This publication makes use of data products from the Two Micron All Sky Survey, which is a joint project of the University of Massachusetts and the Infrared Processing and Analysis Center/California Institute of Technology, funded by the National Aeronautics and Space Administration and the National Science Foundation. The DENIS project has been partly funded by the SCIENCE and the HCM plans of the European Commission under grants CT920791 and CT940627. It is supported by INSU, MEN and CNRS in France, by the State of Baden-Württemberg in Germany, by DGICYT in Spain, by CNR in Italy, by FFwFBWF in Austria, by FAPESP in Brazil, by OTKA grants F-4239 and F-013990 in Hungary, and by the ESO C\&EE grant A-04-046.

\section{References}

Aaronson, M., \& Mould, J. 1980, ApJ, 240, 804

Battinelli, P., \& Demers, S. 2011, A\&A, 525, A69

Bellazzini, M., Correnti, M., Ferraro, F. R., et al. 2006, A\&A, 446, L1

Bertin, E., \& Arnouts, S. 1996, A\&AS, 117, 393

Catchpole, R. M., \& Feast, M. W. 1973, MNRAS, 164, 11

Demers, S., \& Kunkel, W. E. 1979, PASP, 91, 761

Epchtein, N., de Batz, B., Capoani, L., et al. 1997, Messenger, 87, 27

Feast, M. W. 2009, in AGB Stars and Related Phenomena, eds. T. Ueta, N. Matsunaga, \& Y. Ita, 48 [arXiv: astro-ph/0812.0250]

Feast, M., \& Whitelock, P. 2000, in The Evolution of the Milky Way: Stars versus Clusters, eds. F. Matteucci, \& F. Giovannelli (Dordrecht: Kluwer), 229

Feast, M. W., Whitelock, P. A., \& Menzies, J. W. 2006, MNRAS, 369, 791

Glass, I. S., \& Lloyd Evans, T. 1981, Nature, 291, 303

Goswami, A., Karinkuzhi, D., \& Shantikumar, N. S. 2010, MNRAS, 402, 1111
Grenon, M., Mattei, J. A., Eyer, L., \& Foster, G. 2000, The Carbon Star Phenomenon, ed. R. F. Wing (Dordrecht: Kluwer), IAU Symp., 177, 171

Habing, H. J., \& Whitelock, P. A. 2004, in Asymptotic Giant Branch Stars, eds. H. J. Habing, \& H. Olofsson (Springer), Chap. 8

Ibata, R., Irwin, M. J., Lewis, G. F., \& Stolte, A. 2001, ApJ, 547, L133

Iben, I., \& Renzini, A. 1983, ARA\&A, 21, 271

Ita, Y., \& Matsunaga, N. 2011, MNRAS, 412, 2345

Kamath, D., Wood, P. R., Soszyński, I., et al. 2010, MNRAS, 408, 522

Koposov, S. E., Belokurov, V., Evans, N. W., et al. 2012, ApJ, 750, 80 Kunder, A., \& Chaboyer, B. 2009, AJ, 137, 4478

Laney, C. D., Joner, M. D., \& Pietrzyński, G. 2012, MNRAS, 419, 1637

Lee, M. G., Freedman, W., Mateo, M., et al. 1993, AJ, 106, 1420

Lorenz, D., Lebzelter, T., Nowotny, W., et al. 2011, A\&A 532, 78

Majewski, S. R., Skrutskie, M. F., Weinberg, M. D., \& Ostheimer, J. C. 2003, ApJ, 599, 1082

Marin-Franch, A., Aparicio, A., Piotto, G., et al. 2009, ApJ, 694, 1498

Mauron, N. 2008, A\&A, 482, 843

Mauron, N., Azzopardi, M., Gogoyan, K., \& Kendall, T. R. 2004, A\&A, 418, 77

Mauron, N., Kendall, T. R., \& Gigoyan, K. 2005, A\&A, 438, 867

Mauron, N., Gigoyan, K. S., \& Kendal, T. R. 2007, A\&A, 475, 843

Menzies, J. W., Whitelock, P. A., Feast, M. W., \& Matsunaga, N. 2010, MNRAS, 406, 86

Menzies, J. W., Feast, M. W., Whitelock, P. A., \& Matsunaga, N. 2011, MNRAS, 414, 3492

Meusinger, H. 2002, IBVS, 5356, 1

Meusinger, H., \& Brunzendorf, J. 2001, IBVS, 5035, 1

Nishida, S., Tanabé, T., Nakada, Y., et al. 2000, MNRAS, 313, 136

Pietrzyński, G., Thompson, I. B., Graczyk, D., et al. 2009, ApJ, 697, 862

Schlegel, D., Finkbeiner, D., \& Davis, M. 1998, ApJ, 500, 525

Soszyński, I., Udalski, A., Szymański, M. K., et al. 2009, Acta Astrom. 59, 239

Stellingwerf, R. F. 1978, ApJ, 224, 953

Totten, E. J., \& Irwin, M. J. 1998, MNRAS, 294, 1

van Loon, J. Th., Marshall, J. R., Matsuura, M., \& Zijlstra, A. A. 2003, MNRAS, 341,1205

Whitelock, P. A. 2012, Astrophys. Space Sci., in press [arXiv: 1201.2997]

Whitelock, P., Menzies, J., Irwin, M. J., \& Feast, M. W. 1999, The Stellar Content of Local Group Galaxies, eds. P. Whitelock, \& R. Cannon (San Francisco: ASP), IAU Symp., 192, 136

Whitelock, P. A., Feast, M. W., \& van Leeuwen, F. 2008, MNRAS, 386, 313

Whitelock, P. A., Menzies, J. W., Feast, M. W., et al. 2009, MNRAS, 394, 795 